Article

\title{
A Novel Role of Eruca sativa Mill. (Rocket) Extract: Antiplatelet (NF-kB Inhibition) and Antithrombotic Activities
}

\author{
Eduardo Fuentes ${ }^{1,2}$, Marcelo Alarcón ${ }^{1,2}$, Manuel Fuentes ${ }^{1}$, Gilda Carrasco ${ }^{3, *}$ and Iván Palomo ${ }^{1,2, *}$ \\ 1 Department of Clinical Biochemistry and Immunohematology, Faculty of Health Sciences, \\ Interdisciplinary Excellence Research Program on Healthy Aging (PIEI-ES), Universidad de Talca, \\ Talca 3460000, Chile; E-Mails: edfuentes@utalca.cl (E.F.); malarcon@utalca.cl (M.A.); \\ mfuentes@alumnos.utalca.cl (M.F.) \\ 2 Centro de Estudios en Alimentos Procesados (CEAP), CONICYT-Regional, Gore Maule 3460000, \\ R09I2001, Chile \\ 3 Horticulture Department, Faculty of Agricultural Sciences, Universidad de Talca, Talca 3460000, Chile \\ * Authors to whom correspondence should be addressed; E-Mails: gcarrasc@utalca.cl (G.C.); \\ ipalomo@utalca.cl (I.P.); Tel.: +56-71-200484; Fax: +56-71-200488.
}

Received: 29 July 2014; in revised form: 24 November 2014 / Accepted: 25 November 2014 / Published: 12 December 2014

\begin{abstract}
Background: Epidemiological studies have shown the prevention of cardiovascular diseases through the regular consumption of vegetables. Eruca sativa Mill., commonly known as rocket, is a leafy vegetable that has anti-inflammatory activity. However, its antiplatelet and antithrombotic activities have not been described. Methods: Eruca sativa Mill. aqueous extract ( 0.1 to $1 \mathrm{mg} / \mathrm{mL})$, was evaluated on human platelets: (i) P-selectin expression by flow cytometry; (ii) platelet aggregation induced by ADP, collagen and arachidonic acid; (iii) IL-1 $\beta$, TGF- $\beta 1$, CCL5 and thromboxane B2 release; and (iv) activation of $\mathrm{NF}-\kappa \mathrm{B}$ and PKA by western blot. Furthermore, (v) antithrombotic activity (200 mg/kg) and (vi) bleeding time in murine models were evaluated. Results: Eruca sativa Mill. aqueous extract (0.1 to $1 \mathrm{mg} / \mathrm{mL}$ ) inhibited P-selectin expression and platelet aggregation induced by ADP. The release of platelet inflammatory mediators (IL-1 $\beta$, TGF- $\beta 1$, CCL5 and thromboxane B2) induced by ADP was inhibited by Eruca sativa Mill. aqueous extract. Furthermore, Eruca sativa Mill. aqueous extract inhibited NF-kB activation. Finally, in murine models, Eruca sativa Mill. aqueous extract showed significant antithrombotic activity and a slight effect on bleeding time. Conclusion: Eruca sativa Mill. presents antiplatelet and antithrombotic activity.
\end{abstract}


Keywords: Eruca sativa Mill.; rocket; antiplatelet; antithrombotic; platelet inflammatory mediators; functional food

\section{Introduction}

Commonly found in the Mediterranean diet, the rocket species is from the family, Brassicaceae. The species includes Eruca sativa Mill., Diplotaxis species and Bunias orientalis, which are eaten at different ontogenic stages [1]. The positive and beneficial effects of the phytochemicals contained in rocket on human health have been reported by several clinical research studies. Moreover, the consumption of green leafy vegetables has been associated with a reduced risk of cardiovascular diseases (CVD) [2]. These beneficial effects have been attributed to the range of phytochemicals they contain, including vitamins A and C, flavonoids and glucosinolates, all of which are found in high levels in Brassicaceae crops $[3,4]$.

Eruca sativa Mill. (commonly known as rocket) is a fast-growing and cool-season crop. The levels can be cut after 20 days and are sequentially harvested from re-growth [5]. Rocket is considered an excellent source of antioxidants, as it includes phenolic compounds, carotenoids, glucosinolates and degradation products, such as isothiocyanates [6]. Furthermore, Eruca sativa Mill. possesses anti-secretory, anti-inflammatory, cytoprotective and anti-ulcer activity against experimentally-induced gastric lesions. The anti-ulcer effect is possibly due to prostaglandin-mediated activity and/or through anti-secretory and antioxidant properties $[7,8]$.

Platelet adhesion, activation and aggregation on the exposed subendothelial extracellular matrix are essential for hemostasis, but may also lead to thrombosis [9]. Platelet activation triggers the synthesis and release of several autocrine and paracrine mediators, including adenosine diphosphate (ADP), thrombin, epinephrine and thromboxane A2 (TXA2) [10,11]. These agonists activate multiple protein-mediated signaling pathways, inducing platelet shape change, degranulation and integrin activation [12]. Hence, platelet activation is the initial and central event leading to atherothrombosis after the rupture of atheromatous plaques [13].

Despite the important role of platelet activation in the development of acute thrombosis and CVD, no data are available regarding the effect of Eruca sativa Mill. on platelet activation and thrombus formation. The aim of this study was to investigate the antiplatelet and antithrombotic activities of aqueous extract from rocket.

\section{Experimental Section}

\subsection{Reagents and Antibodies}

The agonists ADP and arachidonic acid (AA), acetylsalicylic acid (ASA), rose bengal and prostaglandin $\mathrm{E}_{1}$ (PGE1) were obtained from Sigma-Aldrich (St. Louis, MO, USA), and collagen was obtained from Hormon-Chemie (Munich, Germany). Dimethyl sulfoxide (DMSO), HEPES, methanol and anti-phospho-NF-кB p65 were obtained from Sigma-Aldrich (St. Louis, Missouri/MO, USA). Anti-phospho-protein kinase A (PKA) antibody was obtained from Santa Cruz (Biotechnology, 
California, CA, USA). Anti $\gamma$-tubulin monoclonal antibody (4D11) was obtained from Thermo Scientific (Thermo Scientific, Pierce, Rockford, IL, USA). Antibodies (anti-CD62P-PE and anti-CD61-FITC) were obtained from BD Pharmingen (BD Biosciences, San Diego, CA, USA). DMSO 0.2\% was employed as the vehicle for the preparation of working solutions of Eruca sativa Mill. extract.

\subsection{Processing Material}

Eruca sativa Mill. cv. Sauvage leaves were harvested from a crop obtained from a commercial hydroponic company in the Region of Maule, Chile, 25 days after sowing. The float Speedling system was used to grow this crop $[14,15]$.

\subsection{Preparation of Extract}

Extract from Eruca sativa Mill. was obtained according to Fuentes et al. [16]. In brief, the samples were comminuted in a blender and mixed with water:methanol, $7: 3 \mathrm{v} / \mathrm{v}$, then sonicated and centrifuged for $10 \mathrm{~min}$ at $700 \times \mathrm{g}$. Then, the supernatant was lyophilized at $-45^{\circ} \mathrm{C}$ (freeze dried) and stored at $-80{ }^{\circ} \mathrm{C}$ until use.

\subsection{Preparation of Human Platelet Suspensions}

After receiving written informed consent, venous blood samples were taken from six young healthy volunteers. The protocol was authorized by the ethics committee of the Universidad de Talca in accordance with the Declaration of Helsinki (approved by the 18th World Medical Assembly in Helsinki, Finland, 1964). The samples were placed in $3.2 \%$ citrate tubes $(9: 1 \mathrm{v} / \mathrm{v})$ by phlebotomy with a vacuum tube system (Becton Dickinson Vacutainer Systems, Franklin Lakes, NJ, USA). Samples obtained from each volunteer were processed independently for each assay and centrifuged (DCS-16 Centrifugal Presvac RV) at $240 \times g$ for 10 min to obtain platelet-rich plasma (PRP). Subsequently, two-thirds of PRP were removed and centrifuged $(10 \mathrm{~min}$ at $650 \times \mathrm{g})$. The pellet was then washed with HEPES-Tyrode's buffer containing PGE 1 (120 nmol/L). Washed platelets were prepared in HEPES-Tyrode's buffer at a concentration of $200 \times 10^{9}$ platelets/L (Bayer Advia 60 Hematology System, Tarrytown, NY, USA). After blood samples were taken, platelets were kept at $4{ }^{\circ} \mathrm{C}$ during all of the isolation steps.

\subsection{Flow Cytometry Analysis for P-Selectin}

P-selectin expression on platelet surface was analyzed by flow cytometry [17]. Briefly, $480 \mu \mathrm{L}$ of washed platelets were pre-incubated with $20 \mu \mathrm{L}$ of vehicle (DMSO 0.2\%) or Eruca sativa Mill. extract ( 0.1 to $1 \mathrm{mg} / \mathrm{mL}$ ) for $3 \mathrm{~min}$, followed by 6 minutes of stimulation at $37^{\circ} \mathrm{C}$ with ADP $8 \mu \mathrm{mol} / \mathrm{L}$. To determine platelet P-selectin expression, $50 \mu \mathrm{L}$ of the sample were mixed with saturated concentrations of anti-CD62P-PE and anti-CD61-FITC and incubated for $25 \mathrm{~min}$ in the dark. Samples were then acquired and analyzed in an Accuri C6 flow cytometer (BD, Biosciences, San Diego, CA, USA). Platelet populations were gated on cell size using forward scatter (FSC) vs. side scatter (SSC) and CD61 positivity to distinguish them from electronic noise. The light scatter and fluorescence channels were set at logarithmic gain, and 5000 events per sample were analyzed. Fluorescence intensities of differentially- 
stained populations were expressed as the mean channel value using the BD Accuri C6 Software (BD Biosciences, San Diego, CA, USA). All measurements were performed from six separate platelet donors.

\subsection{Measurement of Platelet Aggregation}

Platelet aggregation was monitored by light transmission according to Born and Cross [18], using a lumi-aggregometer (Chrono-Log, Havertown, PA, USA). Briefly, $480 \mu \mathrm{L}$ of PRP in the reaction vessel were pre-incubated with $20 \mu \mathrm{L}$ of vehicle (DMSO $0.2 \%$ ) or Eruca sativa Mill. extract ( 0.1 to $1 \mathrm{mg} / \mathrm{mL}$ ). After $3 \mathrm{~min}$ of incubation, $20 \mu \mathrm{L}$ of agonist (ADP $8 \mu \mathrm{mol} / \mathrm{L}$, collagen $1.5 \mu \mathrm{g} / \mathrm{mL}$ or AA $1 \mathrm{mmol} / \mathrm{L}$ ) were added to initiate platelet aggregation, which was measured for 6 min. The platelet aggregation (maximal amplitude (\%)) was determined by AGGRO/LINK software (Chrono-Log, Havertown, PA, USA). The inhibition of the maximal platelet aggregation was expressed as a percentage with respect to control (DMSO 0.2\%). The concentration required to inhibit platelet aggregation by $50 \%$ (IC50) was calculated from the dose-response curves. All measurements were performed from six separate platelet donors.

\subsection{Measurement of Thromboxane B2, CCL5, TGF-1 $\beta$ and IL-1 $\beta$ Levels}

Thromboxane B2, CCL5, TGF-1 $\beta$ and IL- $1 \beta$ levels were determined using human Quantikine ELISA kits (R\&D systems, Minneapolis, MN, USA). Briefly, washed platelets $\left(200 \times 10^{9}\right.$ platelets/L) were pretreated with vehicle (DMSO $0.2 \%$ ) or Eruca sativa Mill. extract ( 0.1 to $1 \mathrm{mg} / \mathrm{mL}$ ) for $15 \mathrm{~min}$ at $37{ }^{\circ} \mathrm{C}$ and then stimulated by ADP $(8 \mu \mathrm{mol} / \mathrm{L})$ for $15 \mathrm{~min}$ at $37^{\circ} \mathrm{C}$. Finally, the supernatants were collected after centrifugation at $11,000 \times \mathrm{g}$ for $10 \mathrm{~min}$ at $4{ }^{\circ} \mathrm{C}$ and stored at $-70{ }^{\circ} \mathrm{C}$ until use. The thromboxane B2, CCL5, TGF- $1 \beta$ and IL-1 $\beta$ levels of the supernatants were measured using ELISA kits. All measurements were performed from six separate platelet donors.

\subsection{Measurement of cAMP Levels in Human Platelets}

The effect of Eruca sativa Mill. extract $(0.1$ to $1 \mathrm{mg} / \mathrm{mL})$ on cAMP platelet levels was evaluated in $480 \mu \mathrm{L}$ of washed platelets $\left(200 \times 10^{9}\right.$ platelets/L) after a 5 -min incubation period without stirring. The platelet reaction was stopped in ice-cold $15 \%$ trichloroacetic acid, and precipitated proteins were removed by centrifugation. Samples were stored at $-70^{\circ} \mathrm{C}$ until analysis. Before determination, samples were dissolved in $200 \mu \mathrm{L}$ PBS at pH 6.2. The cAMP Parameter Assay Kit (R\&D Systems, Minneapolis, MN, USA) was used. All measurements were performed from six separate platelet donors.

\subsection{Western Blotting}

Washed platelets $\left(200 \times 10^{9}\right.$ platelets/L) were pre-incubated with vehicle (DMSO $\left.0.2 \%\right)$ or Eruca sativa Mill. extract ( 0.1 to $1 \mathrm{mg} / \mathrm{mL})$ for $3 \mathrm{~min}$ and activated with ADP $(8 \mu \mathrm{mol} / \mathrm{L})$ for $6 \mathrm{~min}$. Then, platelets were lysed with $0.2 \mathrm{~mL}$ of lysis buffer in ice for $30 \mathrm{~min}$ and heated for $10 \mathrm{~min}$ at $95{ }^{\circ} \mathrm{C}$. Equal quantities of total protein $(30 \mu \mathrm{g})$ were subjected to SDS-PAGE under reducing conditions and transferred to a nitrocellulose membrane. The proteins were detected with anti-phospho-PKA, anti-phospho-NF-кB p65 and anti- $\gamma$-tubulin antibodies. All measurements were performed from six separate platelet donors. 


\subsection{Murine Model of Thrombosis}

This study was carried out under recommendations by the Guide for the Care and Use of Laboratory Animals of the National Institutes of Health. The protocol was approved by the Committee on the Ethics of Animal Experiments of the University of Talca. All efforts were made to minimize suffering. Thrombosis in mice was performed by photochemical injury using modified methods described by Przyklenk and Whittaker [19]. Briefly, C57BL/6 mice (12-16 weeks old) were anesthetized with a combination of tribromoethanol (270 mg/kg) and xylazine (13 mg/kg). Thrombosis was induced by an injection of $50 \mathrm{mg} / \mathrm{kg}$ rose bengal through the tail vein followed by illumination of the exposed mesenteric artery with a 1.5-mW green light laser $(532 \mathrm{~nm})$. Blood flow was monitored for $60 \mathrm{~min}$, and stable occlusion was defined as a blood flow of $0 \mathrm{~mL} / \mathrm{min}$ for $3 \mathrm{~min}$. Eruca sativa Mill. extract (200 mg/kg, $n=6$ ), vehicle (DMSO 0.2\% group, $n=6$ ) and ASA (200 mg/kg, $n=6$ ) were administered intraperitoneally $30 \mathrm{~min}$ before the experiment. After laser exposure, the injury image generated was recorded with a charge-coupled device camera (Lumenera Corporation, Ottawa, ON, Canada). The image was analyzed with ImageJ software (version 1.26t, NIH, USA). The region of interest (vessel occlusion) was defined as the target artery, which included the portion of the target artery that was larger than the maximum injured area. Using software tools, thrombus size was measured in the region of interest. Rectal temperatures were similar and within the physiological range between all experimental animals throughout the experimental period.

\subsection{Bleeding Assay}

C57BL/6 mice were anesthetized with a combination of tribromoethanol ( $270 \mathrm{mg} / \mathrm{kg}$ ) and xylazine $(13 \mathrm{mg} / \mathrm{kg}$ ) and placed prone on a warming pad from which the tail protruded. The same amounts of Eruca sativa Mill. extract (200 mg/kg, $n=6$, intraperitoneally), ASA (200 mg/kg, $n=6$, intraperitoneally) or vehicle (DMSO $0.2 \%, n=6$, intraperitoneally) were given as described in the thrombosis model. An incision was made on the ventral surface of the mice tails about $2 \mathrm{~mm}$ from the tip [20]. The bleeding time was measured in seconds (s) until bleeding stopped.

\subsection{Statistical Analysis}

Data were analyzed using SPSS version 17.0 (SPSS, Inc., Chicago, IL, USA) and expressed as the mean \pm standard error of mean (SEM). Six or more independent experiments were performed for the different assays. Results were expressed as a percentage of inhibition or as a percentage of control (as 100\%). The fifty-percent inhibitory concentration (IC50) of Eruca sativa Mill. extract was calculated from the dose-response curves. Differences between groups were analyzed by a one-way analysis of variance (ANOVA) using Tukey’s post hoc test. $p$-values $<0.05$ were considered significant. 


\section{Results}

\subsection{Effect of Eruca sativa Mill. Extract on Platelet Activation}

The effect of Eruca sativa Mill. extract on P-selectin expression in human platelets after stimulation by ADP in PRP was measured by flow cytometry (Figure 1). In the presence of Eruca sativa Mill. extract $(1 \mathrm{mg} / \mathrm{mL})$, the P-selectin expression was inhibited from $58 \pm 3$ to $45 \pm 3 \%(p<0.05)$.

Figure 1. Effect of Eruca sativa Mill. extract on platelet activation. P-selectin expression was determined by flow cytometry. The basal bar represents the fluorescence in the non-stimulated sample. The graph depicts the mean \pm SEM of $n=6$ experiments. An asterisk (* $p<0.05$ ) denotes a statistically significant difference when Eruca sativa Mill. extract was compared with the activated control analyzed by one-way ANOVA and Tukey's post hoc test.

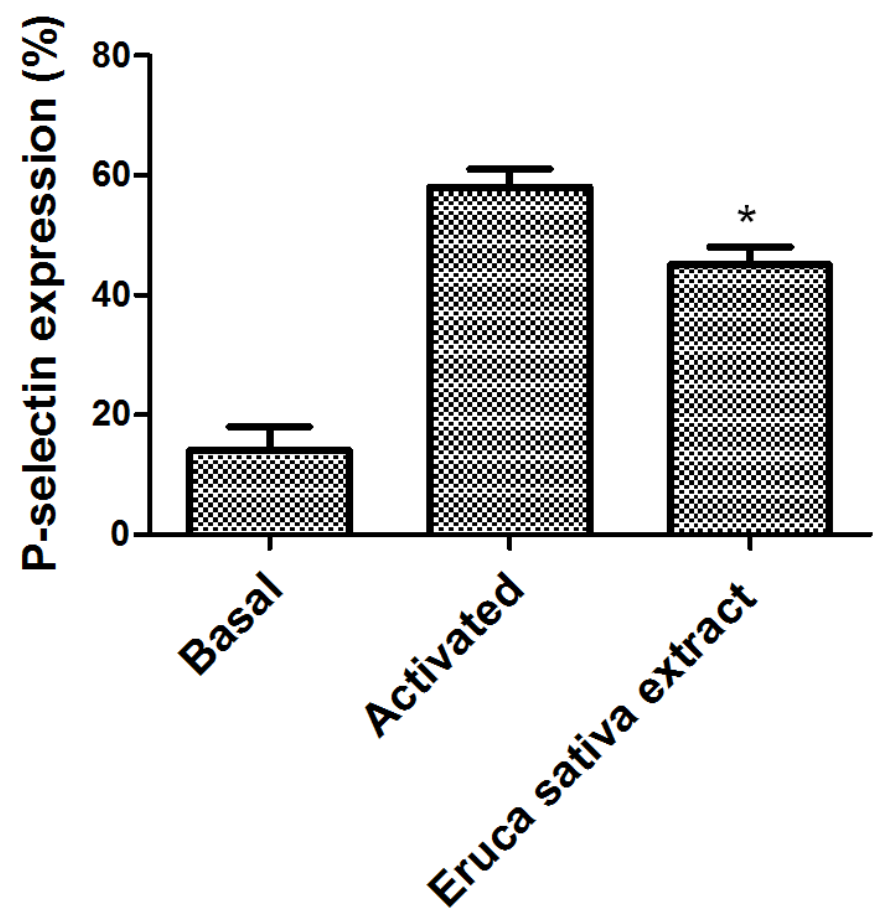

\subsection{Effect of Eruca sativa Mill. Extract on Platelet Aggregation}

The effects of Eruca sativa Mill. extract on platelet aggregation induced by ADP, collagen and AA are shown in Figure 2. Eruca sativa Mill. extract inhibited ADP-induced platelet aggregation with a 50\% inhibitory concentration (IC50) of $0.71 \mathrm{mg} / \mathrm{mL}$. In addition, Eruca sativa Mill. extract only showed a mild inhibitory effect (17 \pm 4 and 16\% $\pm 3 \%, p<0.05)$ over collagen and AA-induced platelet aggregation at a concentration of $1 \mathrm{mg} / \mathrm{mL}$. 
Figure 2. Effects of Eruca sativa Mill. extract on ADP $(8 \mu \mathrm{mol} / \mathrm{L})$, collagen $(1.5 \mu \mathrm{g} / \mathrm{mL})$ and AA (1 mmol/L) induced platelet aggregation. Results were expressed as platelet aggregation (\%) (mean \pm SEM, $n=6$ ). Asterisks (* $p<0.05$ and ${ }^{* * *} p<0.001$ ) denote a statistically significant difference when compared with the activated control analyzed by one-way ANOVA and Tukey's post hoc test. AA = arachidonic acid.
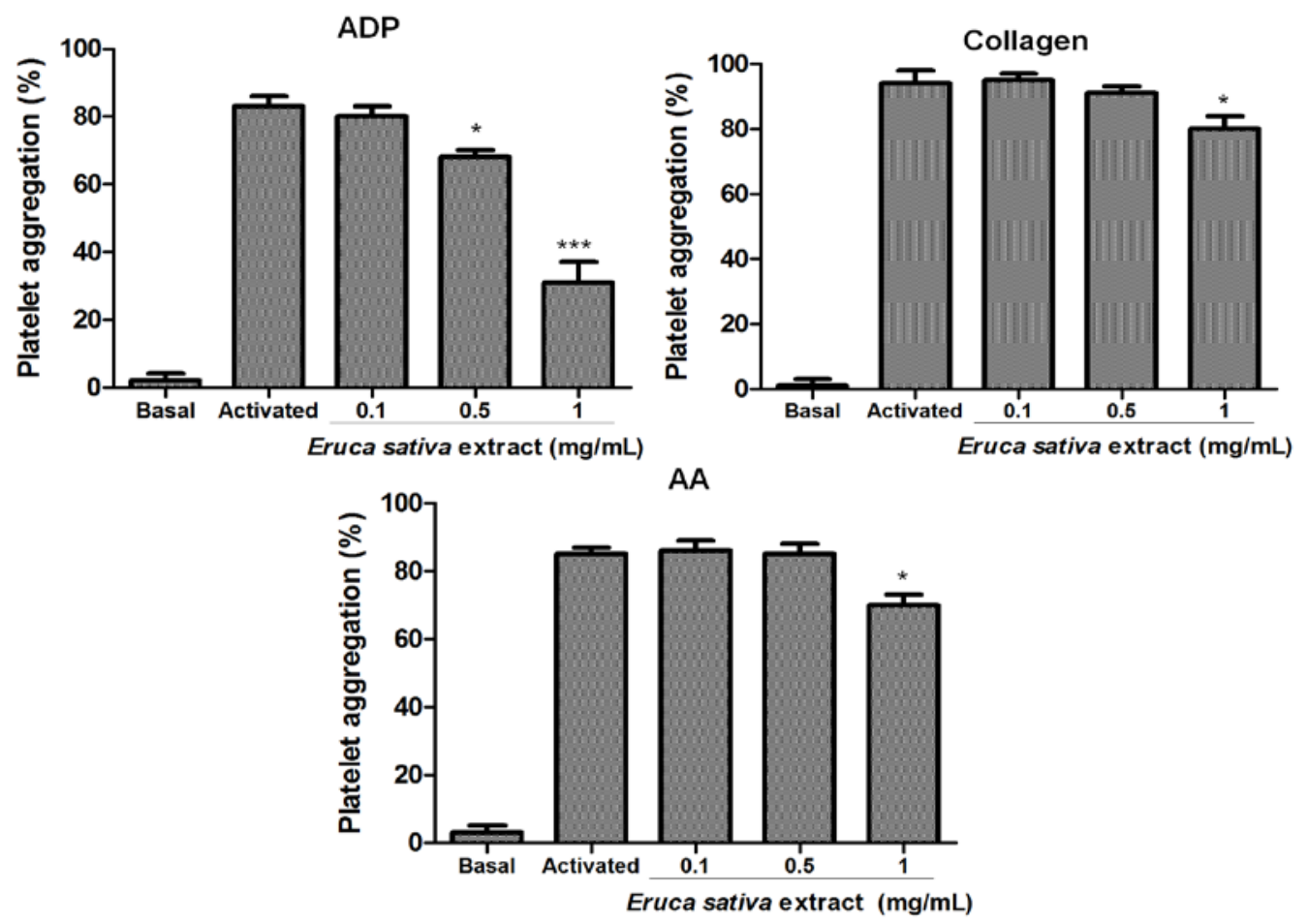

\subsection{Effects of Eruca sativa Mill. Extract on Thromboxane B2, CCL5, TGF-1 $\beta$ and IL-1 Levels}

As shown in Figure 3, resting platelets produced relatively low thromboxane B2, CCL5, TGF-1 $\beta$ and IL-1 $\beta$ levels compared to ADP-activated platelets. Eruca sativa Mill. extract $(0.1$ to $1 \mathrm{mg} / \mathrm{mL}$ ) concentration-dependently inhibited thromboxane B2, CCL5, TGF-1 $\beta$ and IL-1 $\beta$ levels in platelets stimulated by ADP $(8 \mu \mathrm{mol} / \mathrm{L})$.

In washed platelets, ADP-induced thromboxane B2 release in the presence of Eruca sativa Mill. extract at $0.1,0.5$ and $1 \mathrm{mg} / \mathrm{mL}$ was inhibited from $16 \pm 1.9 \mathrm{ng} / \mathrm{mL}$ in the control group to $11 \pm 1$, $10 \pm 1.7$ and $9 \pm 1.3 \mathrm{pg} / \mathrm{mL}(p<0.001$ ), respectively (Figure 3A). In addition, we examined the effect of Eruca sativa Mill. extract on platelet CCL5 release. As observed in Figure 3B, Eruca sativa Mill. extract significantly reduced ADP-induced platelet CCL5 release from $888 \pm 11 \mathrm{pg} / \mathrm{mL}$ in the control group to $780 \pm 9$ and $755 \pm 17 \mathrm{pg} / \mathrm{mL}(p<0.01$ ), at concentrations of 0.5 and $1 \mathrm{mg} / \mathrm{L}$, respectively. Furthermore, in this study, Eruca sativa Mill. extract at $0.1,0.5$ and $1 \mathrm{mg} / \mathrm{mL}$ inhibited the effect of ADP-induced TGF-1 $\beta$ release by $22 \pm 1,38 \pm 2$ and $67 \% \pm 1 \%(p<0.001)$, respectively (Figure 3C). Meanwhile, ADP-induced IL-1 $\beta$ release was inhibited by $26 \pm 3$ and $57 \% \pm 3 \%(p<0.001)$ in the presence of Eruca sativa Mill. extract at 0.5 and $1 \mathrm{mg} / \mathrm{mL}$, respectively (Figure 3D). 
Figure 3. Effects of Eruca sativa Mill. extract on the release of thromboxane $\mathrm{B}_{2}$ (A), CCL5 (B), TGF- $\beta 1$ (C) and IL-1 $\beta$ (D) induced by ADP $(8 \mu \mathrm{mol} / \mathrm{L})$ from human platelets. The graph depicts the mean \pm SEM of $n=6$ experiments. ${ }^{* *} p<0.01$ and ${ }^{* * *} p<0.001$ indicates differences when compared with activated control, analyzed by one-way ANOVA and Tukey's post hoc test.
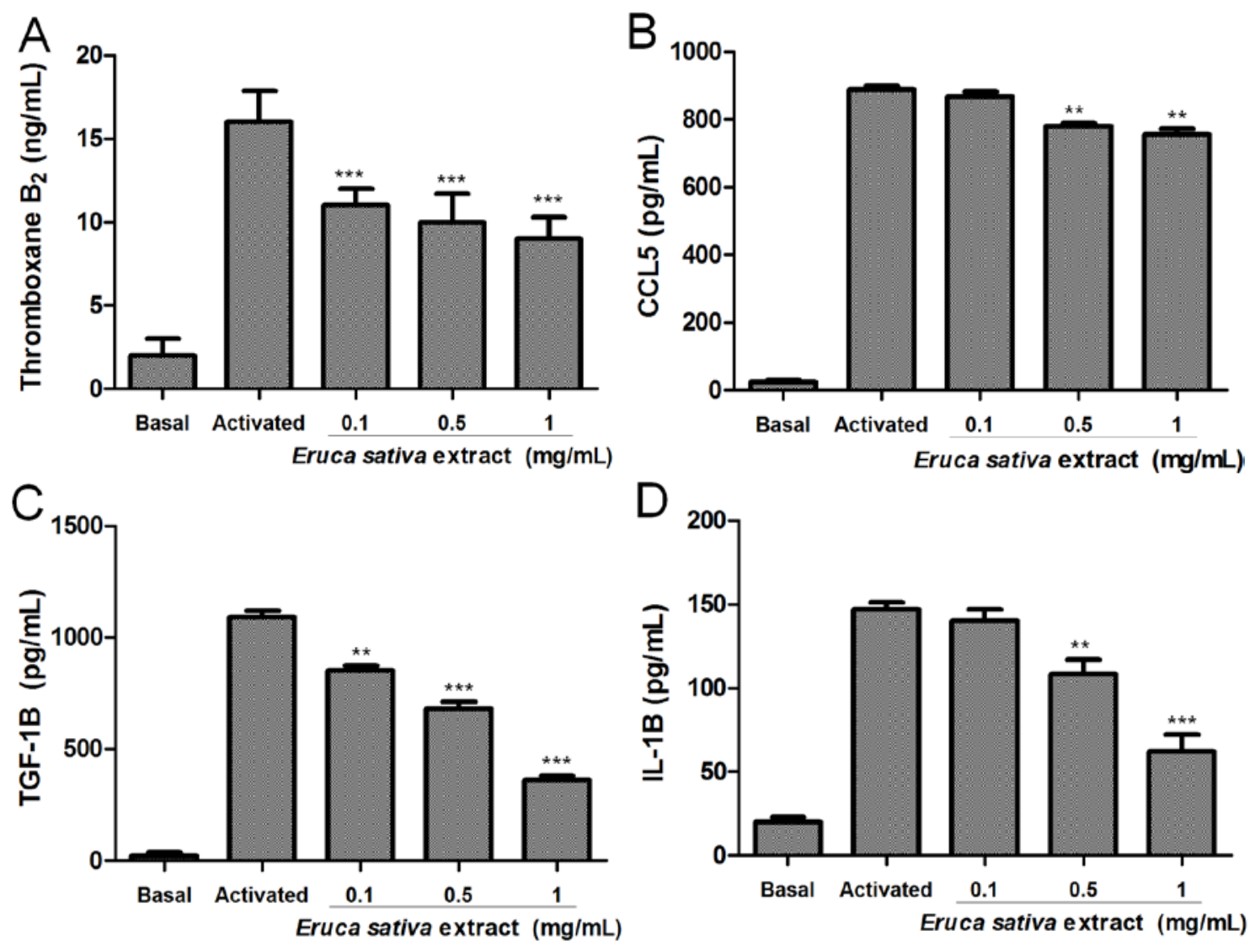

\subsection{Eruca sativa Mill. Extract and Intraplatelet Levels of cAMP}

We investigated whether platelet inhibition by Eruca sativa Mill. extract was mediated by changes of intraplatelet cAMP levels. Eruca sativa Mill. extract $(0.1$ to $1 \mathrm{mg} / \mathrm{mL})$ did not show any effect on intraplatelet levels of cAMP. Levels of cAMP in resting platelets were marked lower than those observed in PGE1 $(0.02 \mathrm{mmol} / \mathrm{L})$-treated platelets $(p<0.001)$.

\subsection{Effects of Eruca sativa Mill. Extract on PKA and NF- $\kappa B$}

PKA activation by cAMP phosphorylates multiple target proteins in numerous platelet inhibitory pathways. Here, the treatment of washed platelets with Eruca sativa Mill. extract (0.1 to $1 \mathrm{mg} / \mathrm{mL})$ did not increase the phosphorylation of PKA (Figure 4).

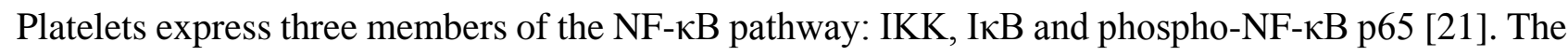
present study demonstrated that p65 phosphorylation was markedly increased in ADP-induced activation of washed platelets, and Eruca sativa Mill. extract (0.1 to $1 \mathrm{mg} / \mathrm{mL})$ concentration-dependently attenuated

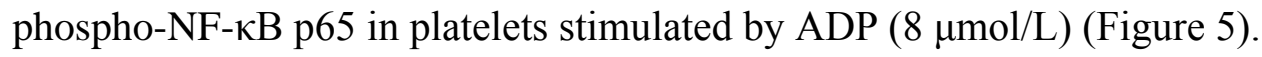


Figure 4. Effect of Eruca sativa Mill. extract on phospho-PKA in activated platelets by ADP ( $8 \mu \mathrm{mol} / \mathrm{L}$ ). The activated group corresponds to prostaglandin E1 (PGE1) plus ADP. Data are presented as the mean \pm SEM of $n=6$ experiments. *** $p<0.001$ indicates the difference between activated and basal groups as analyzed by one-way ANOVA and Tukey's post hoc test.

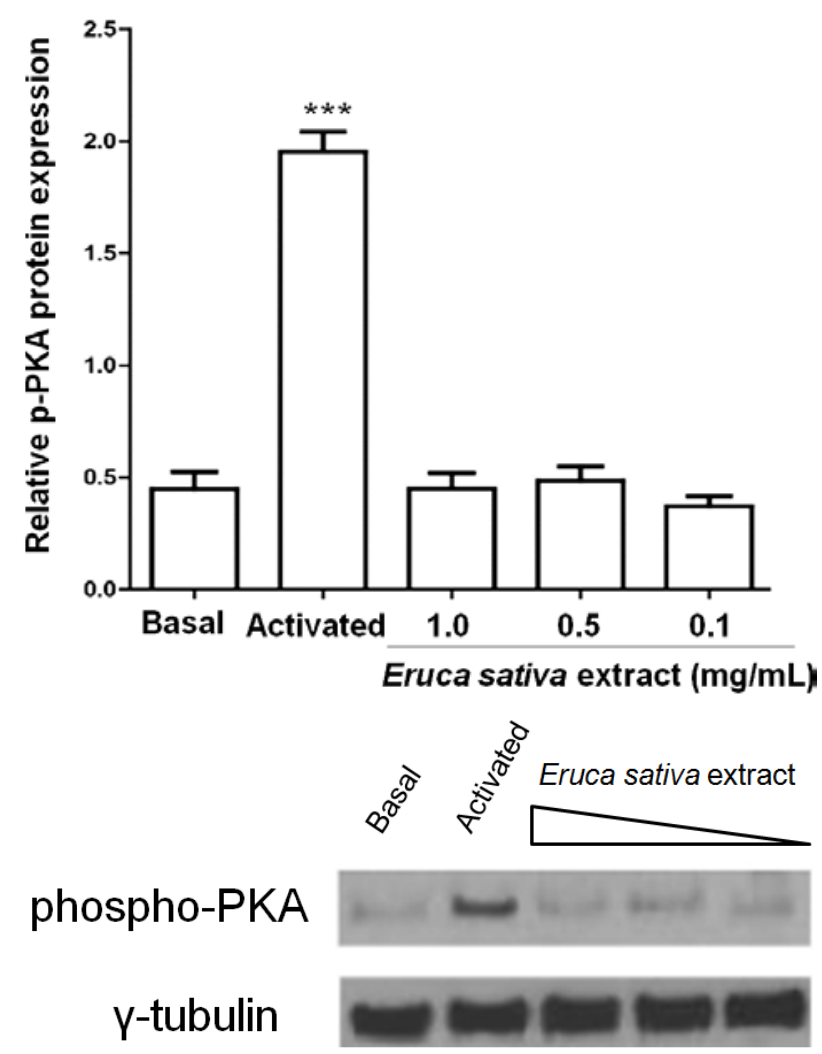

\subsection{Effect of Eruca sativa Mill. Extract on Arterial Thrombus Formation and Bleeding Time}

As shown in Figure 6, the mesenteric artery of untreated mice (control) was completely occluded by a stable bulky thrombus $30 \mathrm{~min}$ after laser injury. In contrast, one intraperitoneal bolus injection of Eruca sativa Mill. extract (200 mg/kg) delayed vessel occlusion to $60 \mathrm{~min}$ and reduced the maximum occlusion (occlusion for $100 \%)$ to $57 \% \pm 2 \%(p<0.01)$.

We measured Eruca sativa Mill. extract-induced C57BL/6 mouse blood loss after tail snip at the same concentration that was used for arterial thrombus formation in vivo $(200 \mathrm{mg} / \mathrm{kg}$, a single bolus intraperitoneally injection). In this study, the same antithrombotic concentration used of Eruca sativa Mill. extract did not cause significant bleeding measured by tail snip. Thus, the bleeding time by Eruca sativa Mill. extract of $187 \pm 19 \mathrm{~s}(n=6)$ was not statistically significantly higher than the control $(171 \pm 26 \mathrm{~s}, n=6)(p>0.05)$.

Concurrently, ASA (200 mg/kg) reduced the maximum occlusion (occlusion for 100\%) to $29 \% \pm 3 \%(p<0.01)$. However, at the same antithrombotic concentration, the bleeding time by ASA of $290 \pm 23 \mathrm{~s}(n=6)$ was statistically significantly higher than the control $(171 \pm 26 \mathrm{~s}, n=6)(p<0.05)$. 
Figure 5. Effect of Eruca sativa Mill. extract on phospho-NF- $\kappa \mathrm{B}$ p65 in activated platelets by $\operatorname{ADP}(8 \mu \mathrm{mol} / \mathrm{L})$. Washed platelets were collected, and subcellular extracts were analyzed for phospho-NF- $\kappa \mathrm{B}$ p65, as described in the Experimental Section. Data are presented as the mean \pm SEM of $n=6$ experiments. ${ }^{* *} p<0.01$ and ${ }^{* * *} p<0.001$ indicates differences between activated and Eruca sativa Mill. extract groups as analyzed by one-way ANOVA and Tukey's post hoc test.
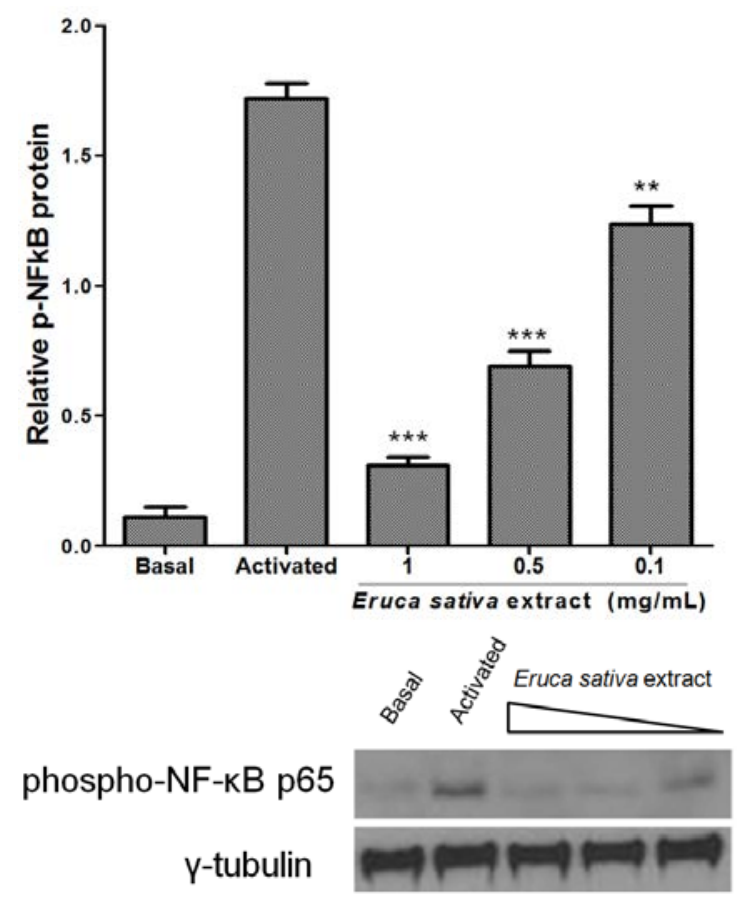

Figure 6. Eruca sativa Mill. extract inhibited arterial thrombosis formation. Representative images of thrombus formation after laser irradiation in the vehicle (DMSO 0.2\%) control group ( $n=6$ ), ASA (acetylsalicylic acid; $200 \mathrm{mg} / \mathrm{kg} ; n=6$ ) and Eruca sativa extract (200 mg/kg, $n=6$ ) to $60 \mathrm{~min}$. Each photograph represents the percentage of occlusions caused by thrombus formation. I, intima; M, media; A, adventitia.

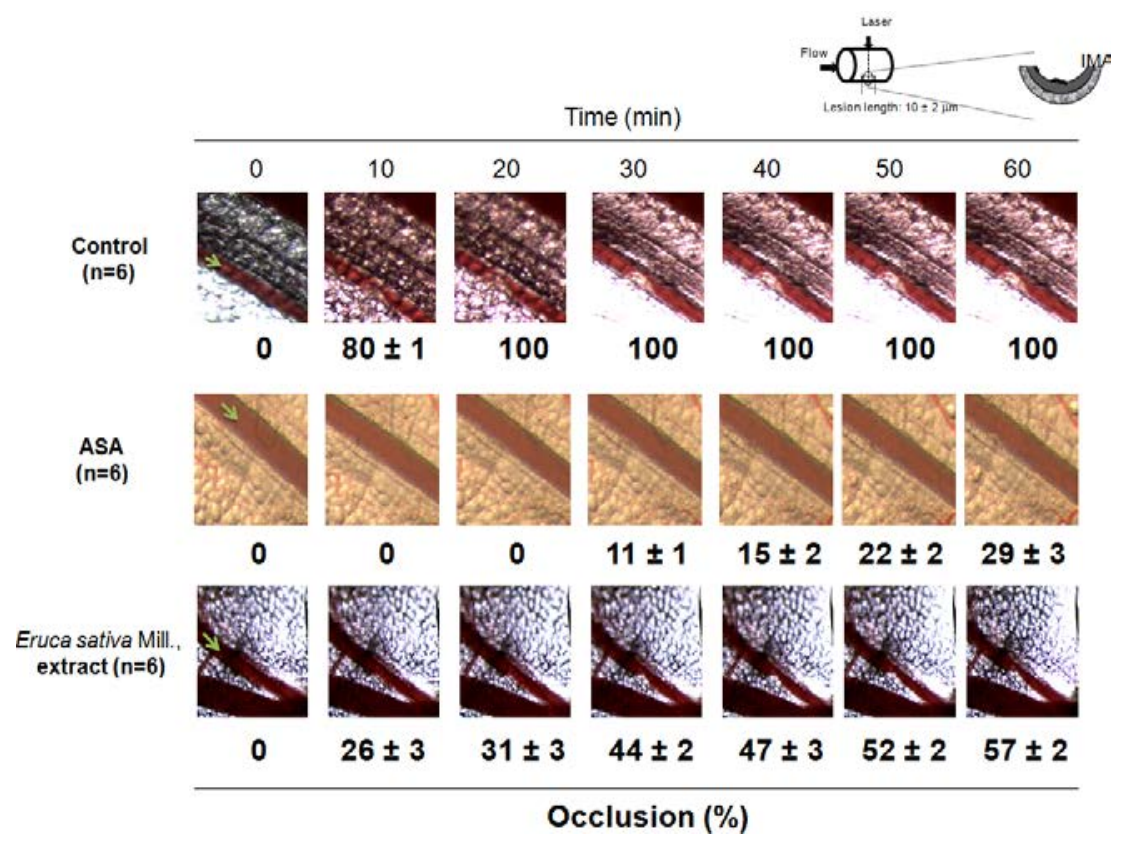




\section{Discussion}

In this study, we demonstrated for the first time that Eruca sativa Mill. extract inhibits platelet aggregation and activation, reduces platelet release of atherosclerotic-related inflammatory mediators (thromboxane B2, CCL5, TGF-1 $\beta$ and IL-1 $\beta$ levels) and decreases in vivo thrombus formation. In addition, evidence is provided that Eruca sativa Mill. extract antiplatelet activities are associated with NF- $\kappa$ B inhibition.

Although antiplatelet drugs have a cardiovascular protective function, many also have side effects (including headaches, gastrointestinal symptoms, skin rash and bleeding) [22]. Therefore, there is an urgent need to identify more effective and safe antiplatelet and antithrombotic agents. In this way, herbs, medicinal plants, spices and vegetables are a potential source to help combat various diseases, including CVD. In recent years, Eruca sativa Mill. rocket leaves, a member of the Brassicaceae family, have been eaten (at different ontogenic stages) all over the world. The leaves are eaten raw or cooked, and Eruca sativa Mill. flowers are also consumed [23,24]. The seeds, roots, leaves and flowers of Eruca sativa Mill. contain different flavonoid profiles [1]. Kaempferol derivatives represent the major group of phenolics present in Eruca sativa Mill. leaves (77\%-88\% of total phenolics), followed by quercetin and isorhamnetin-3,4-diglucoside, representing $9 \%$ and $16.3 \%$ of the total phenolics, respectively [25,26]. The antiplatelet activity of Eruca sativa Mill., could be by the presence of kaempferol, quercetin and isorhamnetin [27-29].

In this study, Eruca sativa Mill. extract displayed in vitro and in vivo antiplatelet activities. Thus, Eruca sativa Mill. extract significantly inhibits platelet activation (less P-selectin expression) and platelet aggregation induced by ADP and showed only a low inhibition over collagen and AA.

Platelet inflammatory mediators (thromboxane B2, CCL5, TGF-1 $\beta$ and IL-1 $\beta$ ) contribute to atherosclerotic lesion development and arterial thrombogenesis [30]. These results show that Eruca sativa Mill. extract inhibited human platelet thromboxane B2, TGF-1 $\beta$ and IL-1 $\beta$ levels induced by ADP. Furthermore, to a lesser extent, Eruca sativa Mill. extract inhibited human platelet CCL5 levels induced by ADP.

Although platelets are anucleated cells, they express several transcription factors that exert non-genomic functions (i.e., NF- $\kappa \mathrm{B}$ ) [31]. Platelet activation triggers $\mathrm{I} \kappa \mathrm{B} \alpha$ phosphorylation and

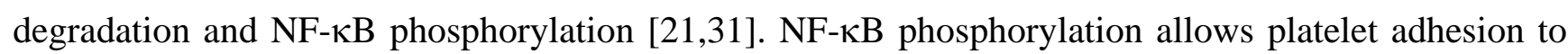
fibrinogen, glycoprotein IIb/IIIa activation, P-selectin expression and TxA2 formation, which was also demonstrated [31]. In the study, Eruca sativa Mill. extract markedly inhibited NF- $\kappa \mathrm{B}$ activation in ADP-stimulated platelets.

Using a murine model of real-time thrombus formation [32], we demonstrated, for the first time, that Eruca sativa Mill. extract prevented thrombus growth in vivo. Moreover, antiplatelet drugs that are currently available inevitably increase bleeding risk at antithrombotic doses [33]. This study showed that Eruca sativa Mill. extract possesses antithrombotic efficacy without significant bleeding. 


\section{Conclusions}

Rocket extract shows antiplatelet activity (inhibition of platelet activation, aggregation and release of inflammatory mediators), and the mechanism of action could be by NF- $\mathrm{B}$ inhibition. However, further studies are needed to expand Eruca sativa Mill. extract properties in the setting of the NF- $\mathrm{B}$ pathway.

\section{Acknowledgements}

This work was funded by the CONICYT REGIONAL/GORE MAULE/CEAP/R09I2001, Interdisciplinary Excellence Research Program on Healthy Aging (PIEI-ES) and supported by Grant No. 1130216 (I.P., M.G., R.M., M.A., J.C.) and No. 11140142 (E.F.) from Fondecyt, Chile.

\section{Author Contributions}

Conceived and designed the experiments: E.F.; M.A. and I.P. Performed the experiments: E.F.; M.A. and M.F. Contributed reagents/materials/analysis tools: I.P. and G.C. Contributed to the writing of the manuscript: E.F.; G.C. and I.P.

\section{Conflict of Interest}

The authors declare no conflicts of interest.

\section{References}

1. Bennett, R.N.; Rosa, E.A.; Mellon, F.A.; Kroon, P.A. Ontogenic profiling of glucosinolates, flavonoids, and other secondary metabolites in Eruca sativa (salad rocket), Diplotaxis erucoides (wall rocket), Diplotaxis tenuifolia (wild rocket), and Bunias orientalis (Turkish rocket). J. Agric. Food Chem. 2006, 54, 4005-4015.

2. Joshipura, K.J.; Hu, F.B.; Manson, J.E.; Stampfer, M.J.; Rimm, E.B.; Speizer, F.E.; Colditz, G.; Ascherio, A.; Rosner, B.; Spiegelman, D.; et al. The effect of fruit and vegetable intake on risk for coronary heart disease. Ann. Intern. Med. 2001, 134, 1106-1114.

3. Jin, J.; Koroleva, O.A.; Gibson, T.; Swanston, J.; Magan, J.; Zhang, Y.; Rowland, I.R.; Wagstaff, C. Analysis of phytochemical composition and chemoprotective capacity of rocket (Eruca sativa and Diplotaxis tenuifolia) leafy salad following cultivation in different environments. J. Agric. Food Chem. 2009, 57, 5227-5234.

4. Bell, L.; Wagstaff, C. Glucosinolates, myrosinase hydrolysis products, and flavonols found in rocket (Eruca sativa and Diplotaxis tenuifolia). J. Agric. Food Chem. 2014, 62, 4481-4492.

5. Jakse, M.; Hacin, J.; Kacjan, N. Production of rocket (Eruca sativa Mill.) on plug trays and on a floating system in relation to reduced nitrate content. Acta Agric. Slov. 2013, 101, 59-68.

6. Villatoro-Pulido, M.; Font, R.; Saha, S.; Obregon-Cano, S.; Anter, J.; Munoz-Serrano, A.; De Haro-Bailon, A.; Alonso-Moraga, A.; Del Rio-Celestino, M. In vivo biological activity of rocket extracts (Eruca vesicaria subsp. sativa (Miller) Thell) and sulforaphane. Food Chem. Toxicol. 2012, 50, 1384-1392. 
7. Alqasoumi, S.; Al-Sohaibani, M.; Al-Howiriny, T.; Al-Yahya, M.; Rafatullah, S. Rocket “Eruca sativa”: A salad herb with potential gastric anti-ulcer activity. World J. Gastroenterol. 2009, 15, 1958-1965.

8. Khan, H.; Khan, M.A. Antiulcer Effect of Extract/Fractions of Eruca sativa : Attenuation of Urease Activity. J. Evid. Based Complement. Altern. Med. 2014, 19, 176-180.

9. Nieswandt, B.; Pleines, I.; Bender, M. Platelet adhesion and activation mechanisms in arterial thrombosis and ischaemic stroke. J. Thromb. Haemost. 2011, 9 (Suppl. 1), 92-104.

10. Li, Z.; Delaney, M.K.; O’Brien, K.A.; Du, X. Signaling during platelet adhesion and activation. Arterioscler. Thromb. Vasc. Biol. 2010, 30, 2341-2349.

11. Li, Z.; Zhang, G.; Le Breton, G.C.; Gao, X.; Malik, A.B.; Du, X. Two waves of platelet secretion induced by thromboxane A2 receptor and a critical role for phosphoinositide 3-kinases. J. Biol. Chem. 2003, 278, 30725-30731.

12. Offermanns, S. Activation of platelet function through G protein-coupled receptors. Circ. Res. 2006, 99, 1293-1304.

13. Penz, S.; Reininger, A.J.; Brandl, R.; Goyal, P.; Rabie, T.; Bernlochner, I.; Rother, E.; Goetz, C.; Engelmann, B.; Smethurst, P.A.; et al. Human atheromatous plaques stimulate thrombus formation by activating platelet glycoprotein VI. FASEB J. 2005, 19, 898-909.

14. Carrasco, G. Semilleros en sistema flotante. In Tratado de Cultivos sin Suelo; Urresterazu, M., Ed.; Ediciones Mundi Prensa: Madrid, España, 2004; p. 914.

15. Carrasco, G.; Izquierdo, J. Almaciguera flotante para la producción de almácigos hortícolas. In Manual Técnico; Universidad de Talca y FAO Oficina Regional para América Latina y El Caribe, Universidad de Talca: Talca, Chile, 2005.

16. Fuentes, E.; Castro, R.; Astudillo, L.; Carrasco, G.; Alarcón, M.; Gutierrez, M.; Palomo, I. Bioassay-guided isolation and HPLC determination of bioactive compound that relate to the anti-platelet activity (adhesion, secretion and aggregation) from Solanum lycopersicum. Evid. Based Complement. Altern. Med. 2012, 2012, 1-10.

17. Frojmovic, M.; Wong, T.; van de Ven, T. Dynamic measurements of the platelet membrane glycoprotein IIb-IIIa receptor for fibrinogen by flow cytometry. I. Methodology, theory and results for two distinct activators. Biophys. J. 1991, 59, 815-827.

18. Born, G.V.; Cross, M.J. The Aggregation of Blood Platelets. J. Physiol. 1963, 168, 178-195.

19. Przyklenk, K.; Whittaker, P. Adaptation of a photochemical method to initiate recurrent platelet-mediated thrombosis in small animals. Lasers Med. Sci. 2007, 22, 42-45.

20. De Clerck, F.; Goossens, J.; Reneman, R. Effects of anti-inflammatory, anticoagulant and vasoactive compounds on tail bleeding time, whole blood coagulation time and platelet retention by glass beads in rats. Thromb. Res. 1976, 8, 179-193.

21. Liu, F.; Morris, S.; Epps, J.; Carroll, R. Demonstration of an activation regulated NF-kappaB/ I-kappaBalpha complex in human platelets. Thromb. Res. 2002, 106, 199-203.

22. Michelson, A.D. Advances in antiplatelet therapy. Hematol. Am. Soc. Hematol. Educ. Program 2011, 2011, 62-69.

23. Cavaiuolo, M.; Ferrante, A. Nitrates and glucosinolates as strong determinants of the nutritional quality in rocket leafy salads. Nutrients 2014, 6, 1519-1538. 
24. Lamy, E.; Schroder, J.; Paulus, S.; Brenk, P.; Stahl, T.; Mersch-Sundermann, V. Antigenotoxic properties of Eruca sativa (rocket plant), erucin and erysolin in human hepatoma (HepG2) cells towards benzo(a)pyrene and their mode of action. Food Chem. Toxicol. 2008, 46, 2415-2421.

25. Pasini, F.; Verardo, V.; Cerretani, L.; Caboni, M.F.; D’Antuono, L.F. Rocket salad (Diplotaxis and Eruca spp.) sensory analysis and relation with glucosinolate and phenolic content. J. Sci. Food Agric. 2011, 91, 2858-2864.

26. Weckerle, B.; Michel, K.; Balazs, B.; Schreier, P.; Toth, G. Quercetin 3,3',4'-tri-O-be ta-D-glucopyranosides from leaves of Eruca sativa (Mill.). Phytochemistry 2001, 57, 547-551.

27. Tzeng, S.H.; Ko, W.C.; Ko, F.N.; Teng, C.M. Inhibition of platelet aggregation by some flavonoids. Thromb. Res. 1991, 64, 91-100.

28. Sempinska, E.; Kostka, B.; Krolikowska, M.; Kalisiak, E. Effect of flavonoids on the platelet adhesiveness in repeatedly bred rats. Pol. J. Pharmacol. Pharm. 1977, 29, 7-10.

29. Mosawy, S.; Jackson, D.E.; Woodman, O.L.; Linden, M.D. Treatment with quercetin and 3',4'-dihydroxyflavonol inhibits platelet function and reduces thrombus formation in vivo. J. Thromb. Thromb. 2013, 36, 50-57.

30. Ferroni, P.; Basili, S.; Davi, G. Platelet activation, inflammatory mediators and hypercholesterolemia. Curr. Vasc. Pharmacol. 2003, 1, 157-169.

31. Malaver, E.; Romaniuk, M.A.; D’Atri, L.P.; Pozner, R.G.; Negrotto, S.; Benzadon, R.; Schattner, M. NF-kappaB inhibitors impair platelet activation responses. J. Thromb. Haemost. 2009, 7 , 1333-1343.

32. Fukuoka, T.; Hattori, K.; Maruyama, H.; Hirayama, M.; Tanahashi, N. Laser-induced thrombus formation in mouse brain microvasculature: effect of clopidogrel. J. Thromb. Thrombolysis 2012, 34, 193-198.

33. Serebruany, V.L.; Malinin, A.I.; Eisert, R.M.; Sane, D.C. Risk of bleeding complications with antiplatelet agents: Meta-analysis of 338,191 patients enrolled in 50 randomized controlled trials. Am. J. Hematol. 2004, 75, 40-47.

(C) 2014 by the authors; licensee MDPI, Basel, Switzerland. This article is an open access article distributed under the terms and conditions of the Creative Commons Attribution license (http://creativecommons.org/licenses/by/4.0/). 